\title{
Phronesis
}

\section{La compétence à lire et acter les finalités effectives de l'école dans un dispositif partenarial École-Communauté comme condition de réalisation de l'intervention socio-éducative Competence to read and put in act the actual finalities of the school in a partnership curriculum between School and Community as a condition of achieving socio-educational intervention}

\author{
Yves Couturier, Chantal Lefebvre, Angèle Bilodeau et Robert Bastien
}

Volume 2, numéro 2-3, avril-juillet 2013

Intervention et pratique éducative : Reflet et/ou tensions entre instruction, socialisation et qualifications?

URI : https://id.erudit.org/iderudit/1018071ar

DOI : https://doi.org/10.7202/1018071ar

Aller au sommaire du numéro

Éditeur(s)

Institut de recherche sur les pratiques éducatives

ISSN

1925-4873 (numérique)

Découvrir la revue

Citer cet article

Couturier, Y., Lefebvre, C., Bilodeau, A. \& Bastien, R. (2013). La compétence à

lire et acter les finalités effectives de l'école dans un dispositif partenarial

École-Communauté comme condition de réalisation de l'intervention

socio-éducative. Phronesis, 2(2-3), 36-42. https://doi.org/10.7202/1018071ar

\section{Résumé de l'article}

Cet article porte sur une initiative de collaboration École-Communauté dans le contexte montréalais. L'initiative Un milieu ouvert sur ses écoles (MOÉ) présente toutes les caractéristiques d'une action à base communautaire reprenant, selon une composition cependant fort particulière, l'ensemble des ingrédients connus d'une collaboration École-Communauté efficiente. Nous montrerons dans cet article en quoi cette composition produit par petites touches une innovation, dont l'essence s'exprime tout entière par le renversement symbolique énoncé dans le nom même de l'initiative, soit le fait que ce soit la communauté qui s'ouvre à l'école, et non le contraire, comme c'est très souvent le cas. Les résultats que nous présentons découlent d'une analyse qualitative réalisée sur un corpus de données documentaires, d'entrevues et de notes d'observation visant à modéliser la pratique professionnelle qui incarne le plus concrètement l'intervention de MOÉ, soit celle des intervenants communautaires scolaires (ICS). 


\title{
La compétence à lire et acter les finalités effectives de l'école dans un dispositif partena- rial École-Communauté comme condition de réalisation de l'intervention socio-éducative
}

\author{
Yves COUTURIER ${ }^{\star}$, Chantal LEFEBVRE**, Angèle BILODEAU ${ }^{\star * *}$ et Robert BASTIEN ${ }^{\star * * *}$
}

\section{${ }^{\star}$ IUGS}

Université de Sherbrooke 1036, rue Belvédère Sud

Sherbrooke (QC)

Canada, J1H 4C4

Yves.couturier@usherbrooke.ca

\author{
${ }^{* *}$ CSSS-Centre affilié universi- ${ }^{* * *}$ Direction de santé publique de \\ taire de Bordeaux-Cartierville-St- l'ASSSM \\ Laurent \\ 1301 Sherbrooke Est \\ Montréal (QC) \\ Canada
}

\author{
${ }^{* * * * \text { IRSP }}$ \\ Université de Montréal \\ 1301 rue Sherbrooke Est \\ Montréal (Québec) H2L 1M3 \\ rbastien@santepub-mtl.qc.ca
}

Mots-clés : école-communauté; intervention socio-éducative-partenariat

Résumé : Cet article porte sur une initiative de collaboration École-Communauté dans le contexte montréalais. L'initiative Un milieu ouvert sur ses écoles (MOÉ) présente toutes les caractéristiques d'une action à base communautaire reprenant, selon une composition cependant fort particulière, l'ensemble des ingrédients connus d'une collaboration École-Communauté efficiente. Nous montrerons dans cet article en quoi cette composition produit par petites touches une innovation, dont l'essence sexexprime tout entière par le renversement symbolique énoncé dans le nom même de l'initiative, soit le fait que ce soit la communauté qui souvre à lécole, et non le contraire, comme ceest très souvent le cas. Les résultats que nous présentons découlent d’une analyse qualitative réalisée sur un corpus de données documentaires, d'entrevues et de notes d’observation visant à modéliser la pratique professionnelle qui incarne le plus concrètement l'intervention de MOÉ, soit celle des intervenants communautaires scolaires (ICS).

Title: Competence to read and put in act the actual finalities of the school in a partnership curriculum between School and Community as a condition of achieving socio-educational intervention

\section{Key-words : school-community; socio-educative intervention; partnership}

Abstract: This article focuses on a collaborative school-community in the city of Montreal. The initiative An open its middle schools (EOM) has all the characteristics of a community-based work resuming, according to a very particular composition, however, all known efficient collaboration School-Community ingredients. We show in this paper how this product innovation by small touches composition, which is expressed by the whole gas contained in the very name of the initiative symbolic reversal, is the fact that it is the community that sopener at school, and not the contrary, as is often the case. The results we present the result of a qualitative analysis carried out on a corpus of documentary, interviews and observation notes to model professional practice which embodies the practical intervention EOM, that of community stakeholders school (ICS). 


\section{Introduction}

\section{La collaboration Communauté-École à partir de lélève}

L'implantation du Programme de formation de l'école québécoise constitue une tentative d’effectuer un virage socio-constructiviste dans la façon de concevoir léducation au Québec (Lenoir, Larose, Lessard, 2005). Cette évolution fondamentale sur le plan épistémologique (au moins en principe) est également soutenue par des configurations sociales appelant la modification même de la mission de lécole, dont on lui demande de plus en plus de jouer un rôle accru en matière de socialisation (Lenoir, 2010) ou déducation à la santé (Murray et al., 2007), par exemple. En fait, l'école est de plus en plus considérée comme un lieu privilégié et efficace pour atteindre les jeunes à propos d’objets historiquement peu ou pas scolaires (World Health Organization, 2003). Cet élargissement de la mission scolaire a pour effet la formulation d’appels multiples à la collaboration intersectorielle pour accroître la capacité scolaire à soutenir la réussite éducative des élèves (Deslandes et Bertrand, 2001). Ces appels sont sous-tendus d'une conception très étendue de la réussite éducative, postulant ontologiquement des continuités fortes dans l'expérience de l'enfant, notamment la continuité scolaire-parascolaire-hors scolaire, et la continuité Famille-Communauté-École (Sheldon et Van Voorhis, 2004). Dans ce contexte, le ministère de l'Éducation, du Loisir et du Sport du Québec invite les acteurs scolaires à s'ouvrir à la communauté (MEQ, 2000) pour créer une école plus sensible à la continuité Famille-Communauté-École, et ce, tant sur le plan des savoirs que sur celui des diverses missions de lécole en vue. De telles continuités rendent lécole plus efficace à rencontrer les attentes sociales en matière de socialisation et d'instruction (Epstein, 1995; 2005). Cet appel a conduit à la mise en place de quantité de mesures aux niveaux ministériels et scolaires, notamment soutenus par la ratification de l'entente de complémentarité liant le ministère de l'Éducation, du Loisir et du Sport, ainsi que le ministère de la Santé et des Services sociaux (MELS, 2003), mais aussi nombre d'initiatives locales (Boulanger et al., sous presse).

Cest dans ce contexte que s'est développé l'initiative Un milieu ouvert sur ses écoles (MOÉ), une action intersectorielle développée en 2001 par la Table de concertation jeunesse Bordeaux-Cartierville. Cette table regroupe une diversité d'acteurs provenant des secteurs municipal, scolaire, sécurité publique, santé et communautaire, qui constatèrent une désaffiliation relative d'une part de la jeunesse de leur territoire, fortement pluriethnique et défavorisée sur le plan socio-économique. L'initiative MOÉ a pris la forme concrète de la mise à disposition d'un intervenant communautaire scolaire (ICS) à temps plein dans chacune des écoles du territoire (n=7). L'ICS a pour fonction première de développer avec et autour de chacune des écoles un réseau cohérent de collaborations et d’activités visant à favoriser les liens FamilleCommunauté-École. Son action seffectue localement et de façon collective, et vise, par le tissage de liens sociaux et la transformation de la dynamique Famille-Communauté-École, la « capacitation » des acteurs qui agissent dans ce système (Jourdan, 2010). Les ICS élaborent puis réalisent une programmation organisée autour de six axes d'intervention :

1) faire évoluer la vie parascolaire en fonction des besoins des élèves, de leurs familles, et de la communauté;

2) favoriser l'intégration des communautés ethno-culturelles;

3) offrir une continuité d'intervention dans les divers cycles temporels de lélève;

4) mobiliser les parents;

5) créer des liens entre les ordres d'enseignement primaire et secondaire et entre les écoles du quartier;

6) favoriser l'amélioration de la santé et du bien-être des jeunes.

1.

\section{Cadre théorique et méthodologie}

Cet article présente les résultats d'une recherche qualitative et partenariale réalisée de 2006 à 2009 visant à décrire la pratique des ICS ${ }^{1}$. Les résultats découlent d'une analyse réalisée sur un corpus de données documentaires, d'entrevues et d'observations, et d'un journal de bord rédigé par les ICS. Des rencontres avec l'ensemble de l'équipe MOÉ (intervenants et direction) ont permis une validation partenariale des primo-résultats. En outre de décrire la pratique, la recherche visait également à analyser l'initiative au regard de sa prétention quant à son

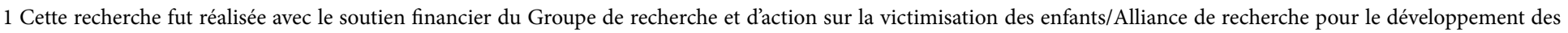

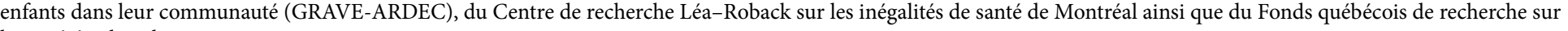
la société et la culture.
} 
caractère réputé innovant. Nous avons donc travaillé à mieux comprendre ce qui pouvait être affirmé comme innovateur dans la pratique observée.

Sur le plan théorique, létude s'inspire de la théorie de la structuration de Giddens articulant les concepts d'action, de structure et de sens. Ce triptyque conceptuel conçoit la pratique sociale comme une production d'actions dans un contexte traversé de structures qui conditionnent partiellement l'action. Mais selon cette perspective, laction structurée est aussi un lieu de "sens ", appréhendable dans les discours que les praticiens énoncent pour expliciter les buts de leurs actions (Giddens, 1987). Le discours est cependant plus qu'un simple accès au sens, il tend à performer la cohérence des actions (Schwandt, 2005). Dans cette perspective, la capacité réflexive de l'acteur situé est inhérente à la pratique, mais également conditionnée dans le rapport structure-action (Couturier, 2006). Le monde social est alors constamment, mais partiellement, recréé par les acteurs sociaux, dans un processus co-génératif (Bourdieu, 2000). La pratique constitue donc l'analyseur (Lourau, 1971) premier de l'articulation du structurel et du sens.

Sur le plan méthodologique, cette étude sest faite de manière rétrospective et prospective. Dans le mouvement rétrospectif, nous avons documenté les actions et les réseaux d'acteurs à partir d'une analyse documentaire. Dans le mouvement prospectif, nous avons poursuivi l’analyse documentaire de documents produits pendant la fenêtre d’observation. Nous avons également effectué une observation ethnographique de séquences d'actions. Pour ce faire, nous avons pris une posture semi-participative en faisant le choix de questionner les acteurs pendant le cours de leur action (tout en minimisant les interférences). Cela nous a permis d’accéder aux finalités visées, aux valeurs sous-jacentes, aux connaissances mobilisées, et à des éléments de contexte hors du champ immédiat de l’observation; il sagissait par ce type d’observation de rendre intelligible un ensemble déléments de la pratique caractérisés par une plus faible dicibilité (Dodier, 1993). L’observation a été réalisée avec l'appui d'une grille d’observation ouverte, mais reprenant les catégories générales du cadre théorique. Nous avons également effectué des entretiens compréhensifs auprès du coordinateur de MOÉ, des deux directions décole, de professionnels scolaires, et de partenaires de la communauté). Les collectes de données ont été réalisées dans deux écoles, l'une primaire et l'autre secondaire. Au total, 85 documents ont été analysés (360p.), 118h d’observation, cinq entretiens individuels et deux entrevues collectives ont été réalisés. De plus, deux rencontres de réflexivité entre les chercheurs et l'ensemble de léquipe MOÉ ont été effectuées pour assurer une forme de validité écologique. Les données collectées ont été saisies dans le logiciel de soutien à l'analyse qualitative Atlas.ti. Elles ont ensuite été codifiées en fonction des dimensions du cadre théorique et de certains paramètres émergents du corpus. Des opérations de co-codage pour une validation inter-juges ont aussi été réalisées. Tout au long de la codification, la rédaction de mémos analytiques a permis de mettre en forme lanalyse à partir des verbes d’action. À partir de ces données, la dimension processuelle de la pratique a été reconstruite, conduisant à dégager cinq formes de la pratique.

\section{La pratique de l'intervenant communautaire-scolaire}

Nous avons reconstruit cinq formes d'action de l'ICS. Nous verrons pour chacune de ces formes ce qui la caractérise de façon à pouvoir, dans un second temps, apprécier son caractère innovant, le cas échéant.

\section{1 Être présent dans le milieu}

Agir auprès des élèves, là où ils se trouvent, appelle une modalité d'intervention de présence proximale requérant une grande mobilité dans lécole. Pour faire son œuvre, cette mobilité doit être en phase avec les temps et mouvements réels des élèves. Cette forme d'intervention, proche des stratégies de reaching out (aller là où se trouve l'usager potentiel des services), requiert également des gestes de visibilité qui permettront la sollicitation directe de l'ICS par les élèves. Par exemple, l'ICS installe son bureau dans un espace ouvert aux jeunes (ex. : le café étudiant) de façon à voir et être vu. Au-delà de son bureau officiel, l'ICS doit aussi se faire facilement repérable autant dans l'espace scolaire vécu, soit l'école en tant que telle avec ses alentours, que dans les espaces transitionnels entre elle et la maison ou les autres lieux de vie des élèves. Pour leur être pleinement accessible, l'ICS doit notamment déployer une présence adaptée au calendrier des scolaires. Par exemple, la flexibilité de son horaire et de ses conditions de travail lui permet de rencontrer les élèves sur l'heure du midi ou après les cours, mais aussi la fin de semaine, lors de journées pédagogiques et même pendant la période estivale selon les besoins de l'intervention qu'il vise. La modalité de présence à lélève est donc plus souple et plus informelle que celles des enseignants ou des intervenants réguliers des services complémentaires, ce qui lui permet d’observer de façon plus globale les interactions des élèves entre eux. Surtout au primaire, sa présence dans l'école permet de rencontrer et d'échanger avec les parents sur un mode à première vue banal. Il saisira chaque occasion pour annoncer une activité ou solliciter la participation des élèves ou de leur famille. Sa présence dans les événements et fêtes de quartier lui permet aussi de prendre contact avec les familles, mais hors de l’espace institué (et instituant) quest lécole. Cela importe particulièrement pour les familles socio-économiquement défavorisées ou d'immigration récente.

L'ICS doit donc posséder un heixis (Bourdieu, 1980) lisible pour tout élève, soit une façon dêttre inscrite dans le corps, à travers la façon de se vêtir, de bouger, de parler. Il se distingue aussi des autres intervenants scolaires par sa façon d’occuper l'espace, par sa manière dêttre, par ses gestes professionnels ostensiblement réceptifs aux élèves. Cette visibilité lui permet dêtre interpellé, en cas de besoin, sur un mode banal plutôt qu'interventionnel. Écrite plus précisément, sa présence s'effectue d'abord en mode ludique plutôt que clinique, ce qui importe pour tout jeune, et davantage encore pour ceux d’entre eux qui ont une expérience négative des services sociaux ou dont la famille est portée à se méfier des services publics, notamment celles provenant de pays où l'État est davantage une menace qu'une ressource. L'approche des jeunes se fait 
donc avant tout sur un mode amical, voire taquin, le plus souvent sur un ton dédramatisé. La présence d'un ICS aux activités parascolaires en mode ludique permet de tisser des liens de confiance avec les élèves et d'intervenir au besoin. Ainsi, les ICS ne signifient pas aux jeunes ou à leur famille qu'ils sont des cliniciens de l'intervention socio-éducative. Ils se présentent plutôt comme des généralistes du lien social. Le travail sur le lien social est d’ailleurs en phase avec le projet de favoriser l'intégration des familles migrantes à la société québécoise. Pour cela, il peut les mettre en lien avec d'autres parents ou les mobiliser afin qu'ils s'engagent dans la vie scolaire et communautaire.

Cette forme d'action est clairement issue des stratégies classiques de l'animation sociale et du travail de milieu. En ce sens, au seul regard de cette forme d'action, il est difficile de soutenir que MOÉ constitue une innovation dans le champ socio-éducatif.

\section{2 Éduquer}

Certes avant tout ludique, l'intervention de l'ICS se présente explicitement, voire ostensiblement comme socio-éducative et en forte continuité avec la mission de socialisation de lécole, mais aussi avec celle d'instruction (bien qu'indirectement le plus souvent). L'intention éducative de l'ICS s'arrime à celui des autres intervenants socio-éducatifs, plus clairement scolaires, et au projet prioritairement éducatif des enseignants. En fait, les interventions des uns et des autres sont fédérées par la réussite (sans doute relative) du concept de réussite éducative pour structurer les diverses formes d'intervention en contexte scolaire. Bien entendu, cela peut se lire à travers la tension montrée par Lenoir (2010) entre instruction et socialisation. Et sans doute est-il possible de soutenir que cela exprime la surdétermination actuelle de la dimension socialisatrice dans cette tension. Mais force est de constater que le concept fait une œuvre en ordonnançant davantage intervention éducative et intervention socio-éducative (Couturier, Larose et Bédard, 2009).

L'intervention ludique de l'ICS cherche constamment à transférer divers savoirs, savoirs-être et savoirs-faire, qui se situent généralement à la périphérie du curriculum strictement académique, mais qui tendent à le prolonger, et, ce faisant, à réaliser les continuités évoquées supra. La réussite éducative participe ici d'une conception humaniste de l'éducation où le développement et lépanouissement de l'enfant comme citoyen dépassent la seule acquisition des connaissances, tout en affirmant que l'accès au savoir est émancipateur. Concrètement, l'intervention socio-éducative participe explicitement des domaines généraux du Programme de formation de lécole québécoise que sont l’environnement et la consommation, le vivre ensemble et la citoyenneté, la santé et le bien-être. L'action d’éduquer chez l'ICS concernera par exemple le domaine de la santé, à travers des activités parascolaires en vogue dans le quartier, comme le basketball. La création d'un tournoi de basketball visera explicitement à la fois à occuper les longues soirées d'été, à favoriser l'interconnaissance entre élèves de diverses écoles, parfois même à favoriser une meilleure maîtrise du français à loccasion de la rédaction de publicités, par exemple. L'ICS contribue ainsi à la mission de socialisation des élèves en renforçant chez eux certaines compétences sociales à légard de normes citoyennes valorisées. Lorsqu'il cherche à raviver les normes du vivre ensemble en société, l'ICS encourage le développement d'une gamme d'habiletés sociales comme la politesse ou le respect des autres. Par exemple, il rend possibles des démarches de réparation de leurs actes ayant eu des conséquences négatives sur autrui. Ce faisant, il fait en sorte que les activités se déroulent dans le respect des règlements de l'école, même lors des activités parascolaires. De plus, l'ICS invite les élèves à développer leur esprit citoyen en les incitant à des actions de préservation de l'environnement ou de solidarité, par exemple. Il crée pour ce faire des événements qui sensibilisent les élèves à la solidarité et à l'entraide, tel que la participation de la chorale de l'école à une campagne de financement d'un organisme caritatif du quartier. Cette éducation à la citoyenneté cherche aussi à favoriser louverture sur le monde, par exemple par l'organisation d'activités où les élèves du secondaire viennent raconter une légende de leur pays d’origine à d'autres élèves du primaire. Cela permet à la fois de favoriser la transition primaire-secondaire, de familiariser les enfants à l'altérité culturelle, de les encourager à garder des liens avec leur culture d'origine, tout en leur permettant d'accéder à l'histoire et aux traditions québécoises présentées comme la société d’accueil grâce à l'ajout d'un conte québécois à l'activité.

Cette forme d’action est généralement partagée par l'ensemble des intervenants éducatifs et socio-éducatifs contribuant la plupart du temps à une imbrication étroite de la mission d'instruction et de la mission de socialisation. Au seul regard de cette forme, il est donc difficile de soutenir que MOÉ constitue une innovation. Par contre, lorsque nous considérons le fait que MOÉ est une organisation de type associative formellement hors du champ scolaire, sa compétence ici démontrée à lire les finalités effectives de lécole ne constitue sans doute pas à elle seule une caractéristique innovante, mais peut néanmoins paraître comme une condition favorable à l'innovation.

\subsection{Créer des occasions d'engagement des jeunes}

Découlant des deux autres formes d'action, le travail de l'ICS consiste concrètement à organiser de nombreuses activités parascolaires, sportives, culturelles ou sociales. Mais la cible occupationnelle se subsume très clairement dans le projet de produire un éthos de l'engagement chez les élèves, comme caractéristique fondamentale de de la réussite éducative, mais aussi de la réussite citoyenne. Cette stratégie est en phase avec le point de vue ministériel, pour qui de telles « activités complémentaires à la formation du jeune favorisent le développement de son plein potentiel et [...] offrent de multiples occasions d’engagement» (MELS, $2001: 36$ ). Ces activités visent ultimement à contrer les risques de désengagement de l'élève à légard de l'école, de la famille, voire de la société. De telles occasions d'engagement se constituent le plus souvent par la mise en place d'activités ludiques dans l’enceinte scolaire, mais aussi à la périphérie de lécole, notamment les parcs municipaux du quartier. Si l'ICS doit savoir saisir loccasion, il doit tout autant savoir la créer, voire la planifier. Cet intervenant établit donc une programmation pour chaque école, à partir d'une concertation par école effectuée par le coordonnateur de MOÉ et la direction de l'école. À ce niveau, les priorités d'action seront établies en les harmonisant à la programmation des activités scolaires, mais aussi communautaires du quartier. 
Certaines de ces activités se dérouleront sur le temps de classe, comme ce fut le cas pour un rallye de quartier où les élèves, avec leur professeur titulaire, firent la tournée des organismes communautaires du quartier afin qu'ils les connaissent et puissent s'y référer au besoin. Cependant, le plus souvent l'ICS loge ses activités dans les espaces de transition entre les temps académiques comme les récréations, les dîners, la fin des classes, les soirs, les journées pédagogiques, les fins de semaine, la semaine de relâche et la période estivale. À la demande de la direction d'école, d'enseignants ou d'autres professionnels œuvrant dans l'école, l'ICS participe aussi à des activités de la programmation scolaire, telle la Semaine de léducation physique. On le voit, la nature, la territorialité et la temporalité de l’offre d'activités de MOÉ sont caractérisées par une très grande adaptabilité aux particularités de lécole et aux problématiques mises en priorité lors des rencontres de co-gestion tenues entre la direction de lécole et le coordonnateur de MOÉ.

Parfois travailleur de milieu, parfois travailleur de rue, parfois organisateur communautaire ou animateur social, l'ICS prend la forme professionnelle adéquate à l'atteinte d'un objectif convenu avec lécole. Sur le plan structurel, cela pose l'ICS comme un dispositif intersectoriel, considérant l'enfant et sa famille comme principe intégrateur, comme l'appellent d'ailleurs les savoirs probants en cette matière (ex. : CDC, 2003). Cette dimension structurelle se déploie au moins sur deux plans. Le premier concerne le travail de réseau, tendu vers la périphérie de lécole. L'ICS tisse de tels réseaux d’abord (logiquement et chronologiquement) à partir de l'école par des collaborations avec les autres professionnels des services complémentaires, la direction d'école et les enseignants, et par la participation de l'ICS aux différentes instances et comités de lécole, pensons par exemple au comité « toxicomanie ». Sur un second plan, il occupe des espaces stratégiques sur le plan organisationnel lui permettant de constituer des réseaux intra-scolaires. Par sa présence dans les instances de lécole, l'ICS devient rapidement pour ses partenaires celui par qui une activité (souvent jugée chronophage) devient possible. Ainsi, lorsqu’un enseignant ou un spécialiste pense à réaliser un projet, il se tourne vers l'ICS, comme cette infirmière de l'école qui lui demande de l'aide pour mettre sur pied un Comité santé à travers lequel des activités de promotion des saines habitudes de vie seront réalisées. Il infléchira son action de telle sorte que le plan de l'information pourra inclure le tournoi de basketball évoqué plus haut.

Cette forme d'action est généralement partagée par l'ensemble des intervenants socio-éducatifs internes et externes à lécole. Au seul regard de cette forme, il est donc difficile de soutenir que MOÉ constitue une innovation. Par contre, le travail de l'ICS se distingue quelque peu de celui réalisé par d’autres métiers du lien social, tels que le travailleur de milieu (Dupuy, Simard et Champagne, 2001) ou le médiateur social (Barthélémy, 2004), qui privilégient davantage une intervention de type psychosocial ou de planning social, par sa prédilection pour les activités ludiques. Cependant, lentrée par l’activité ludique est aussi partagée par des intervenants socio-éducatifs internes à lécole, comme le technicien en loisir ou l'animateur de vie sociale et communautaire. Mais cette forme d'action sen distingue par deux caractéristiques chez I'ICS. D'abord, elle est volontairement multi-niveaux, c'est-à-dire qu'elle porte autant sur divers registres d'intervention, parfois proximaux, parfois distaux, aux élèves. Pour cela, une participation aux instances scolaires est requise. Puis elle est volontairement intersectorielle, plus précisément à la jonction du monde scolaire et des organisations diverses de sa communauté d’appartenance. La co-gestion du travail de l'ICS par la direction d'école et le coordonnateur de MOÉ, prolongée par le pilotage de quartier de l'initiative, apparait clairement comme une condition structurelle favorable à l'innovation. Cette condition structurelle crée une sorte de littératie croisée à propos des normes et conventions qui régissent l'action des divers partenaires engagés dans le dispositif partenarial.

\subsection{Soutenir des jeunes ayant des difficultés}

La qualité du lien créé avec les élèves fait de l'ICS un intervenant privilégié par certains d’entre eux connaissant une difficulté psychosociale personnelle ou familiale, économique ou migratoire, ou qui ont des difficultés d'insertion scolaire relatives à la violence ou l'isolement. Son mode de présence et sa connaissance profonde du milieu lui permettent de détecter ce qui ne va pas, de mobiliser les acteurs qui pourront contribuer à la solution, etc. En appui sur une connaissance parfois indirecte d'une situation difficile, il cherche à intégrer les élèves qui en sont affectés dans des activités parascolaires afin de les divertir, les occuper, les responsabiliser et les valoriser. Dans certains cas, il est informé des situations difficiles directement par les jeunes eux-mêmes. Dans ce cas, le lien de confiance établi lors d'activités ludiques lui permettra d'agir directement ou indirectement sur la situation. En raison du contexte interethnique du quartier, l'ICS porte une attention particulière aux élèves des classes d'accueil qui vivent une période transitoire par nature difficile.

En raison de l'ampleur et de la diversité des formes que peut prendre son intervention, le rôle d'ICS se distingue de celui des autres professionnels de lécole agissant davantage en mode mono disciplinaire, parfois en mode pluridisciplinaire lorsqu'un séquençage des actions est requis. Un ICS nous dit qu'un : « technicien en loisir fait des activités de loisir dans un cadre bien défini et plutôt technique. Moi j’utilise les activités, parfois spontanées, comme moyen d’aller plus loin avec le jeune et pour établir un lien de confiance ». Selon une direction décole, ce technicien : " fait les projets que j’appellerais institutionnels, selon la tradition de l'École : bal, collation des grades, sortie à la cabane à sucre [etc.]. L'ICS, son travail, ce sont d'autres projets qui viennent donner de la plus-value à lécole. » Dans ce contexte, la direction de lécole réfère à l'ICS des élèves qui manquent d'intérêt pour la vie scolaire et dont la trajectoire parait périlleuse sur le plan de la réussite éducative ou citoyenne, voire personnelle. Tout en respectant les principes de confidentialité, l'ICS éclaire les intervenants concernés sur certaines situations familiales, leur donnant ainsi une compréhension plus globale des problèmes des enfants. Par exemple, concernant un jeune enfant sans fratrie et dont la mère est gravement malade, l'ICS du primaire écrit : " Je continue à y faire attention et je l'ai invité à la première de l'héritage de Darwin à la Maison Théâtre vendredi soir 20 mai. ». L'ICS a aussi obtenu pour cet enfant une place au camp scout à lété. Par-delà l'accès à des ressources, il cherchera à intégrer un tel enfant dans des activités de bénévolat pour lui permettre d'expérimenter des activités porteuses de sens. Ainsi, il demanda aux enfants d’une même famille, dont la mère est décédée, de venir l'aider dans un service de garderie 
qu'il a organisé pour une conférence donnée aux parents. Selon l'ICS : « ils étaient très contents de s'occuper de plus petits. ». Selon Tourrilhes (2003), ce genre de participation donne du sens à l'engagement.

Cette forme d’action est généralement partagée par tous les intervenants éducatifs, mais surtout socio-éducatifs, la nécessité même de trouver une solution à une situation source de souffrance produisant pour tous et chacun un appel fort à se mobiliser. Au seul regard de cette dimension de la présente forme d'action, il est donc difficile de soutenir que MOÉ constitue une innovation. Par contre, nous constatons que l'ICS détient une importante marge de manœuvre dans sa capacité à inventer des solutions personnalisées au problème identifié. Cette capacité créatrice est essentiellement le produit de la position structurelle que prend et que l'on donne à l'ICS dans l'espace scolaire. De facto pleinement membre de l'équipe-école, bien que symboliquement hors de l'espace scolaire (en raison de son heixis, de son mode de son rattachement à MOÉ, du mode de co-gestion de cette ressource, etc.), l'ICS occupe une position d'intermédiaire lui permettant d'effectuer une double médiation entre l'hors-scolaire et le scolaire. Cela constitue une condition favorable à l'innovation.

\subsection{Relayer et intensifier l'action des partenaires de la communauté}

Cette position structurellement médiatrice permet à l'ICS d'agir structurellement sur la skolé, soit ce principe de suspension des contingences du monde dans la classe qui crée lécole comme un lieu clos et qui tend à mettre à distance le mode extérieur (Bourdieu, 1987). La dernière forme d’action consiste à favoriser l'ouverture de la skolé, mais une ouverture ordonnée aux priorités de l'école. Il s’agira donc de favoriser la présence des partenaires de la communauté dans l'école, et à mettre en place des activités ou évènements dans la communauté, dans l'optique de faciliter par ce moyen l'intégration des familles à lécole et dans le quartier. Pour ce faire, l'ICS travaille avec différents types d'organismes du quartier, qu'ils soient communautaires, publics ou privés. Avec certains d’entre eux s'installent des collaborations bidirectionnelles qui solidifient les rapports intersectoriels. Ces liens de confiance et de réciprocité sont indispensables à lefficacité des réseaux communautaires d'intervention, mais plus spécifiquement à la capacité de l'ICS d’agir dans la skolé. Par sa seule présence, l'ICS est un symbole de l'ouverture mutuelle Communauté-École. Par son action dans lécole, et par le relais qu'il fait des activités communautaires dans lespace scolaire, il fait en sorte que lécole donne une meilleure place aux partenaires de la communauté. Différentes stratégies de mise en lien sont utilisées pour ce faire, tel qu'introduire les organismes et faire la promotion de leurs activités lors dévénements scolaires, comme « un mini salon des organismes du quartier» [ICS du primaire]. En contrepartie, l'ICS rappellera avec rigueur aux partenaires communautaires les principes d'une bonne collaboration dans la skolé. Il rappellera notamment que toute action doit participer au projet de réussite éducative, pour l'essentiel tel que lécole le formule. Louverture est donc bivalente, mais différenciée; elle se fera autant du côté de l'école que de la communauté, mais en convenant que l'école est une grande institution qui porte une mission forte. Dans cette orchestration des rapports Communauté-École, l'ICS doit donc travailler à partir de deux cultures organisationnelles distinctes où, dans la communauté, les rapports sétablissent de manière souple et informelle, alors que dans l'espace scolaire, les rapports sont plus conventionnés, découlant d'un cadre de travail plus rigide et d'une structure de gestion de type bureaucratie professionnelle. L'ICS aborde ainsi son mandat tel un médiateur qui identifie les espaces transactionnels possibles. Concrètement, il se pose en traducteur entre ces deux espaces sémantiques, par exemple en démystifiant les attentes et les façons de faire de chacun.

Nous pensons que cette forme d'action contient une source particulière d'innovation, moins dans le fait d'instituer une forme relativement stable de médiation entre lécole et la communauté, que dans cette fonction médiatrice qui pose l'école non pas comme une organisation plus ou moins tacitement inadéquate qu'il serait souhaitable de transformer, mais bien comme une institution porteuse d'une mission légitime. Le projet de transformation de l'institution scolaire, souvent porté par des partenaires hors de lécole, est convié à se reformuler en un projet de co-transformation. Louverture requise est donc à la fois bivalente, ou mutuelle, mais aussi différenciée, en raison de la reconnaissance par tous de l'ampleur et de la multidimensionnalité du projet de réussite éducative dont l'institution scolaire demeure la principale porteuse.

\section{Conclusion : savoir lire l'école comme une institution, pas seulement comme une organisation}

Ce qui caractérise la valence novatrice de l'initiative MOÉ se situe selon dans une compétence particulière que les promoteurs de l'initiative ont su traduire en modalité organisationnelle efficiente. La compétence au bilinguisme intersectoriel est en effet au cœur du métier d'ICS. La fonction traductrice est des plus délicates, car le traducteur se doit de respecter lordre scolaire, sa mission, son fonctionnement, ses façons de faire, tout en ne s'y dissolvant pas.

L'école constructiviste, quoi qu’on pense de la qualité et de l'effectivité de cette évolution, provoque elle-même une brèche dans la skolé. L'initiative MOÉ, pour Un MILIEU ouvert sur ces écoles, répond à cette ouverture non pas par la tactique médiévale de l'envahissement, mais bien par la contre-ouverture, privilégiant ce que les économistes désignent comme l'équilibre haut, ceest-à-dire une sorte de cercle vertueux où louverture appelle plus d’ouverture. Bien entendu, point d'angélisme (Bilodeau !!) ici. Une telle initiative est fragile. Et elle exige des conditions deffectuation et de pérennisation qui sont difficiles à diffuser : une co-gestion à tous les niveaux (opérationnel, managérial et politique), une intensité de présence qui demande des moyens, une liberté d’action qui peut se buter à lorganisation du travail, une tradition partenariale qui prend du temps à sétablir, etc. Le passage de l'innovation locale à sa large diffusion demeure un important défi, dont les conditions demeurent en grande partie à élaborer. 


\section{Références bibliographiques}

Barthélémy, F. (2004). Médiateur social : Dynamiques de fabrication d’une pratique professionnelle. Esprit critique, 6(3). Repéré à http:// www.espritcritique.org

Boulanger, D., Larose, F., Larivée, S., Minier, P., Couturier, Y., \& Kalubi, J. C. (2011). Travail social et participation parentale dans le contexte du partenariat école-famille-communauté - : mise en perspective autour d'une logique socioculturelle. Service social, 57, 74-95.

Bourdieu, P. (1980). Le sens pratique. Paris : Éd. de minuit.

Bourdieu, P. (1987). Méditations pascalienne. Paris : Dunod.

Bourdieu, P. (2000). Esquisse d’une théorie de la pratique. Paris : Seuil.

Centers for Disease Control and Prevention (2003). Chronic disease prevention: promising practices. Atlanta : Centers for Disease Control and Prevention.

Couturier, Y. (2006). La collaboration entre travailleuses sociales et infirmières. Éléments d'une théorie de l'intervention interdisciplinaire. Paris : L'Harmattan.

Couturier, Y., Larose, F. \& Bédard, J. (2009). Continuités et discontinuités de l'intervention éducative et de l'intervention socioéducative en milieu scolaire défavorisé, Les sciences de l'éducation pour l'Ère nouvelle, 42(4) 93-116.

Couturier, Y. \& Chouinard, I. (2008). La relation est-elle soluble dans la didactique? La relationnalité dans les métiers relationnels comme objet d'une didactique des savoirs professionnels. Dans Y. Lenoir \& P. Pastré (dir.), Didactique professionnelle et didactiques disciplinaires en débat (p. 213-223). Toulouse : Octares.

Couturier, Y., Larose, F. \& Bédard, J. (2009). Continuités et discontinuités de l'intervention éducative et de l'intervention socioéducative en milieu scolaire défavorisé. Les sciences de l'éducation pour l'Ère nouvelle, 42(4) 93-116.

Deslandes, R. \& Bertrand, R. (2001). La création d’une véritable communauté éducative autour de lélève; une intervention cohérente et des services mieux harmonisés. CQRS /MEQ : Rapport de recherche. Récupéré à http://crires.ulaval.ca/system/files/publications/DeslandesBertrand2001.pdf? Language $=$ en

Dodier, N. (1993). L’expertise médicale. Essai de sociologie de lexercice du jugement. Paris : Métailié.

Dupuy, S., Simard, P. \& Champagne, D. (2001). Travail de corridor à lécole La Source. Évaluation, Rouyn-Noranda. Régie régionale de la santé et services sociaux de l'Abitibi-Témiscamingue.

Epstein, J. L. (1995). School/Family/Community Partnerships: Caring For The Children We Share. Phi Delta Kapan, 76, 701-711.

Epstein, J. L. (2005). A case study of the Partnership Schools Comprehensive School Reform (CSR) model. Elementary School Journal, 106(2), 151-170.

Giddens, A. (1987). La construction de la société. Paris : Presses universitaires de France.

Gouvernement du Québec (2000). Prendre le virage du succès. Évaluation d’un projet innovateur de concertation entre lécole et la communauté. Québec : ministère de l'Éducation.

Gouvernement du Québec (2003) Deux réseaux, un objectif: le développement des jeunes: Entente de complémentarité des services entre le réseau de la santé et des services sociaux et le réseau de léducation. Québec : ministère de l'Éducation.

Jourdan D. (2010). Éducation à la santé : Quelle formation pour les enseignants Saint

Kanouté, F. (2002). Les profils d'acculturation délèves issus de l'immigration récente à Montréal. Revue des sciences de l'éducation, 28(1), 171-190.

Lenoir, Y. (2010). La tension instruction-socialisation dans les pratiques québécoises d'enseignement au primaire : vers une dérive thérapeutique? Actes du congrès de l'Actualité de la recherche en éducation et en formation (AREF), Université de Genève. Récupéré du site https:// plone.unige.ch/aref2010/communications-orales/premiers-auteurs-en-1/La\%20tension.pdf

Lenoir, Y., Larose F. \& Lessard C. (2005). Le curriculum de l'enseignement primaire : regards critiques sur ses fondements et ses lignes directrices. Sherbrooke : Éditions du CRP.

Lourau, R. (1971). Analyse institutionnelle et éducation. Paris : L'Épi.

Murray, N. G., Low, B. J., Hollis, C., Cross, A. W. \& Davis, S. M. (2007) Coordinated school health programs and academic achievement : a systematic review of the literature. Journal of school health, 77, 589-600.

Schwandt, T.A. (2005). The Centrality of Practice to Evaluation. American Journal of Evaluation, 26(1), 95-105.

Sheldon, S. B \& Van Voorhis, F. L. (2004). Partnership programs in U.S. schools : Their development and relationship to family involvement outcomes. School Effectiveness and School Improvement, 15(2), 125-148.

Tourrilhes, C. (2003). Des espaces intermédiaires de socialisation créés par des jeunes dans les quartiers en difficultés. Repéré de http://jeuneset-societes.cereq.fr/PDF-RJS2/TOURRILHES.pdf

World Health Organization (2003). Creating an environment for emotional and social well-being: an important responsibility of a healthpromoting and child-friendly school. Repéré de http://www.who.int/school_youth_health/media/en/sch_childfriendly_03.pdf 